\title{
Developable or Not Related to Information Loss
}

\author{
William W. S. Chen ${ }^{1}$ \\ ${ }^{1}$ Department of Statistics, The George Washington University, USA \\ Correspondence: Department of Statistics, The George Washington University, Washington D.C. 20013, USA. \\ E-mail: williamwschen@gmail.com
}

Received: June 8, 2018 Accepted: June 26, 2018 Online Published: July 17, 2018

doi:10.5539/jmr.v10n5p28 URL: https://doi.org/10.5539/jmr.v10n5p28

\begin{abstract}
In this paper we present a lemma and two theorems. These theoretical results will be used to test whether or not a given surface model can be developed. We then choose some examples to demonstrate how to perform these tests. All of these theories and examples are for general purposes, and are not restricted to any particular field. Although all examples are in three-dimensional space, it can be expanded to finite n-dimensional Euclidean spaces. The objective of this paper is to link the relationship between developable surfaces and information loss.
\end{abstract}

Keywords: catenary, catenoid, developable surface, exponential family, first fundamental form, gradient vector, Hessian matrix, implicit function, information loss, n-dimensional vector space, surface of revolution, second order efficiency, second fundamental form

Mathematical Subject Classification: 62E99

\section{Introduction}

Fitting a set of observed data to a preselected model was a fundamental rule used by almost all researchers. For instance, in a clinical trial, Tweedie used the Inverse Gaussian Distribution to study the effect of a drug on its first passage time taken by a jejural biopsy capsule leaving the stomach, to travel from the pylorus through the duodenum and into the jejunum. Working in collaboration with statisticians at the Clinical Cancer Research Institute in Liverpool, Tweedie studied the distribution of survival times for patients who had been treated for cancer, and fitted the Inverse Gaussian distribution with considerable success. He also found that the anti-lognormal and Weibull distributions were poor fits. More information on this study and related studies can be found in Villarroya and Oller (1991) and books by Seshadri (1993) and Tweedie (1945). Additionally, astronomers used the extreme value distribution in evaluating the validity of outlying observations. However, this distribution was also used in a variety of applications related to natural phenomena such as rainfall, floods, wind gusts, air pollution, and corrosion. The early works by Fuller and Griffith on the subject were highly specialized, being in the fields of application, as well as in their methods of physical analysis. This area of research initially attracted the interests of theoretical probability researchers, engineers and hydrologists. Historically, work on extreme value length problems can be dated back to as early as 1709, when Nicolar Bernoulli studied the mean largest distance from the origin with $n$ points lying at random on a straight line of length $t$. The most extensive bibliographies that contain more than 350 references about this distribution can be found in Johnson, Kotz, and Balakrishnan. Among all the fitting models, most researchers ignored a fundamental problem, i.e., the information loss problem. Efron (1975) resolved this issue by defining the curvature of a statistical problem by introducing so-called second-order efficiency. The key to his approach is to show that one-parameter exponential families have very nice properties for estimation, testing, and other inference procedures. The reason is that they can be considered to be "straight lines" through the space of all probability distribution on the sample space. He then used these arbitrary one-parameter families to quantify how close they were "exponential". Efron defines a quantity called "the statistical curvature". He finds that statistical curvature is identically zero for exponential families, and positive for non-exponential families. He then demonstrated that the families with a small curvature enjoy the good properties of exponential families, and a large curvature indicates a breakdown of these properties. Efron concludes that statistical curvature turns out to be closely related to Fisher and Rao's theory of "second-order efficiency". In this paper we extended his proposal to general Euclidean n-dimensional space. We prove one lemma and two theorems for the general purposes to test to determine whether a given model can be developed. First, the form of the surface must be determined, so the correct theorem or lemma can be applied. 


\section{Condition for Developable}

We define our notation as follows:

$$
\begin{aligned}
& I=d x \bullet d x=E d u^{2}+2 F d u \bullet d v+G d v^{2} \\
& I I=-d x \bullet d n=e d u^{2}+2 f d u \bullet d v+g d v^{2}
\end{aligned}
$$

where I is called the first fundamental form, and II the second fundamental form, in which E, F and G are the coefficients of the first fundamental form; and $\mathrm{e}, \mathrm{f}$ and $\mathrm{g}$ are the coefficients of the second fundamental form. Both $\mathrm{N}$ (usually mean normal direction) and $\mathrm{x}$ are surface functions of $\mathrm{u}$ and $\mathrm{v}$, which in turn depend on curve $\mathrm{C}$. If we take the derivative with respective to $\mathrm{N}$ and $\mathrm{x}$, we will get the identities:

$$
\begin{aligned}
& d N=N_{u} d u+N_{v} d v, \\
& d x=x_{u} d u+x_{v} d v .
\end{aligned}
$$

Theorem 2.1 A necessary and sufficient condition that a surface be developable is that the Gaussian Curvature vanishes. We shall prove that $e g-f^{2}=0$ is not only a necessary, but also a sufficient condition for a surface to be developable. For this we appeal to the identity.

$$
\begin{aligned}
& e g-f^{2}=\left(X_{u} N_{u}\right)\left(X_{v} N_{v}\right)-\left(X_{u} N_{v}\right)\left(X_{v} N_{u}\right) \\
& =\left(X_{u} x X_{v}\right)\left(N_{u} x N_{v}\right)=\left(N N_{u} N_{v}\right) \sqrt{E G-F^{2}},
\end{aligned}
$$

Which shows that $e g-f^{2}=0$ is identical to $\left(N N_{u} N_{v}\right)=0$. This can happen either (a) when $N_{u}$ OR $\mathrm{N}_{v}$ vanishes, or (b) when $N_{u}$ is collinear with $\mathrm{N}_{v}\left(\mathrm{~N}\right.$ is perpendicular to $N_{u}$ and $\mathrm{N}_{v}$ ). In case (a), $\mathrm{N}$ depends on only one parameter and the surface is the envelope of a family of $\infty^{1}$ planes, and hence a developable. In case (b), we take as one set of coordinate curves on the surface the asymptotic curves with equation

$$
e d u^{2}+2 f d u d v+g d v^{2}=(\sqrt{e} d u+\sqrt{g} d v)^{2}=0
$$

If these curves are taken as the curves $v=$ constant in the new coordinate system, then

$$
\mathrm{e}=\mathrm{f}=0 \text { or } \mathrm{X}_{\mathrm{u}} N_{u}=X_{v} N_{v}=0 \text { hence } \mathrm{N}_{\mathrm{u}}=0,
$$

which brings us back to case(a), again, a developable surface. We now present an example to illustrate theorem 2.1.

Example 2.1 This is a well-known minimal surface that was first discovered by Swiss mathematician Leonhart Euler in 1744. This surface generated by rotating the catenary, $y=\operatorname{acosh}\left(\frac{v}{a}\right)$, about the v-axis. The surface of revolution is called the catenoid. It can be shown that the catenoid is locally isometric to the helicoid. We now show that the surface is undevelopable.

Let $\mathrm{S}$ be a surface of revolution and let

$$
X(u, v)=(a \cosh v \cos u, a \cosh v \sin u, a v), 0<u<2 \pi,-\infty<v<\infty .
$$

We show that the coefficient of the first fundamental form satisfies 


$$
\begin{gathered}
E=G=a^{2} \cosh { }^{2} v, F=0 . \text { and } \\
X_{u}=(-a \cosh v \sin u, a \cosh v \cos u, 0) \\
X_{v}=(a \sinh v \cos u, a \sinh v \sin u, a) \\
E=X_{u} X_{u}=a^{2} \cosh ^{2} v \sin ^{2} u+a^{2} \cosh ^{2} v \cos ^{2} u \\
=a^{2} \cosh ^{2} v \\
F=X_{u} X_{v}=-a^{2} \cosh v \sin u \sinh v \cos u+a^{2} \cosh v \cos u \sinh v \sin u=0 \\
G=X_{v} X_{v}=a^{2} \sinh ^{2} v \cos ^{2} u+a^{2} \sinh ^{2} v \sin ^{2} u+a^{2} \\
=a^{2}\left(1+\sinh ^{2} v\right)=a^{2} \cosh ^{2} v
\end{gathered}
$$

Equations (2.1) and (2.2) yield

$$
\begin{gathered}
E=G=a^{2} \cosh ^{2} v, F=0, \\
E G-F^{2}=a^{4} \cosh ^{4} v, \sqrt{E G-F^{2}}=a^{2} \cosh ^{2} v . \\
X_{u u}=(-a \cosh v \cos u,-a \cosh v \sin u, 0), \\
X_{u v}=(-a \sinh v \sin u, a \sinh v \cos u, 0), \\
X_{v v}=(a \cosh v \cos u, a \cosh v \sin u, 0),
\end{gathered}
$$

From equations (2.1) and (2.3), we can further derive the coefficient of second fundamental form as follow:

$$
\begin{aligned}
& e=\frac{\left(x_{u u} x_{u} x_{v}\right)}{\sqrt{E G-F^{2}}}=\frac{\left|\begin{array}{lll}
-a \cosh v \cos u & -a \cosh v \sin u & 0 \\
-a \cosh v \sin u & a \cosh v \cos u & 0 \\
a \sinh v \cos u & a \sinh v \sin u & a
\end{array}\right|}{a^{2} \cosh ^{2} v} \\
& =\frac{a\left(-a^{2} \cosh ^{2} v \cos ^{2} u-a^{2} \cosh ^{2} v \sin ^{2} u\right)}{a^{2} \cosh ^{2} v}=\frac{-a^{3} \cosh ^{2} v}{a^{2} \cosh ^{2} v}=-a \\
& f=\frac{\left(x_{u v} x_{u} x_{v}\right)}{\sqrt{E G-F^{2}}}=\frac{\left|\begin{array}{lll}
-a \sinh v \sin u & a \sinh v \cos u & 0 \\
-a \cosh v \sin u & a \cosh v \cos u & 0 \\
a \sinh v \cos u & a \sinh v \sin u & a
\end{array}\right|}{a^{2} \cosh ^{2} v} \\
& =\frac{a\left(-a^{2} \sinh v \sin u \cosh v \cos u+a^{2} \sinh v \cos u \cosh v \sin u\right)}{a^{2} \cosh ^{2} v}=0
\end{aligned}
$$




$$
\begin{aligned}
g & =\frac{\left(x_{v v} x_{u} x_{v}\right)}{\sqrt{E G-F^{2}}}=\frac{\left|\begin{array}{lll}
a \cosh v \cos u & a \cosh v \sin u & 0 \\
-a \cosh v \sin u & a \cosh v \cos u & 0 \\
a \sinh v \cos u & a \sinh v \sin u & a
\end{array}\right|}{a^{2} \cosh ^{2} v} \\
& =\frac{a\left(a^{2} \cosh ^{2} v \cos ^{2} u+a^{2} \cosh ^{2} v \sin ^{2} u\right)}{a^{2} \cosh ^{2} v}=\frac{a^{3} \cosh ^{2} v}{a^{2} \cosh ^{2} v}=a
\end{aligned}
$$

In appendix, the Gaussian Curvature is defined as

$$
K=\frac{e g-f^{2}}{E G-F^{2}}=\frac{-a^{2}}{a^{4} \cosh ^{4} v}=\frac{-1}{a^{2} \cosh ^{4} v}
$$

Even though the Gaussian Curvature is different from zero, $\mathrm{K}$ will decrease to zero, when $\mathrm{v}$ gradually increases to infinity. We may claim that Gaussian Curvature can asymptotically drop to zero, and ignore information loss.

\section{MONGE Patch}

It often occurs that the parametric equation is not available. However, we know the equation $\mathrm{z}=\mathrm{f}(\mathrm{x}, \mathrm{y})$,in other words, we are given $\mathrm{z}$ as a function of $(\mathrm{x}, \mathrm{y})$. Hence, we need to answer the following question: What is the second fundamental form when the surface is given by the equation $\mathrm{z}=\mathrm{f}(\mathrm{x}, \mathrm{y})$ ?

$$
\begin{aligned}
& d z=f_{x} d x+f_{y} d y, \\
& d s^{2}=d x^{2}+d y^{2}+d z^{2}=d x^{2}+d y^{2}+\left(f_{x} d x+f_{y} d y\right)^{2}
\end{aligned}
$$

if we let $p=f_{x}, q=f_{y}$ then

$d s^{2}=\left(1+p^{2}\right) d x^{2}+2 p q d x d y+\left(1+q^{2}\right) d y^{2}$

$$
\begin{array}{ll}
\text { let } E=1+p^{2} F=p q G=1+q^{2} \\
\qquad G-F^{2}=\left(1+p^{2}\right)\left(1+q^{2}\right)-p^{2} q^{2}=1+p^{2}+q^{2} \\
X=(x, y, z=f(x, y)) & X_{x x}=\left(0,0, f_{x x}\right) \\
X_{x}=\left(1,0, f_{x}\right) & X_{x y}=\left(0,0, f_{x y}\right) \\
X_{y}=\left(0,1, f_{y}\right) & X_{y y}=\left(0,0, f_{y y}\right)
\end{array}
$$

if we define $p=z_{x}=f_{x}=\frac{\partial z}{\partial x}$,

$$
\begin{aligned}
& \mathrm{q}=\mathrm{z}_{\mathrm{y}}=f_{y}=\frac{\partial z}{\partial y}, \quad \mathrm{r}=\mathrm{z}_{\mathrm{xx}}=f_{x x}=\frac{\partial^{2} z}{\partial x^{2}}, \\
& \mathrm{~s}=\mathrm{z}_{\mathrm{xy}}=f_{x y}=\frac{\partial^{2} z}{\partial x \partial y}, t=\mathrm{z}_{\mathrm{yy}}=f_{y y}=\frac{\partial^{2} z}{\partial^{2} y},
\end{aligned}
$$




$$
\begin{gathered}
\text { then } e=\frac{\left(x_{x x} x_{x} x_{y}\right)}{\sqrt{E G-F^{2}}}=\frac{\left|\begin{array}{lll}
0 & 0 & f_{x x} \\
1 & 0 & f_{x} \\
0 & 1 & f_{y}
\end{array}\right|}{\sqrt{1+p^{2}+q^{2}}}=\frac{f_{x x}}{\sqrt{1+p^{2}+q^{2}}} \\
f=\frac{\left(x_{x y} x_{x} x_{y}\right)}{\sqrt{E G-F^{2}}}=\frac{\left|\begin{array}{lll}
0 & 0 & f_{x y} \\
1 & 0 & f_{x} \\
0 & 1 & f_{y}
\end{array}\right|}{\sqrt{1+p^{2}+q^{2}}}=\frac{f_{x y}}{\sqrt{1+p^{2}+q^{2}}} \\
g=\frac{\left(x_{y y} x_{x} x_{y}\right)}{\sqrt{E G-F^{2}}}=\frac{\left|\begin{array}{lll}
0 & 0 & f_{y y} \\
1 & 0 & f_{x} \\
0 & 1 & f_{y}
\end{array}\right|}{\sqrt{1+p^{2}+q^{2}}}=\frac{f_{y y}}{\sqrt{1+p^{2}+q^{2}}}
\end{gathered}
$$

When $\mathrm{z}=\mathrm{f}(\mathrm{x}, \mathrm{y})$ the second fundamental form is given by

$$
\begin{aligned}
& I I=e d x^{2}+2 f d x d y+g d y^{2}=\frac{f_{x x} d x^{2}+2 f_{x y} d x d y+f_{y y} d y^{2}}{\sqrt{1+p^{2}+q^{2}}} \\
& =\frac{r d x^{2}+2 s d x d y+t d y^{2}}{\sqrt{1+p^{2}+q^{2}}}
\end{aligned}
$$

In MONGE patch, the Gaussian Curvature can be defined as follow;

$$
K=\frac{f_{x x} f_{y y}-f_{x y}^{2}}{\sqrt{\left(1+p^{2}+q^{2}\right)^{4}}}=\frac{f_{x x} f_{y y}-f_{x y}^{2}}{\left(1+p^{2}+q^{2}\right)^{2}}
$$

We can derive the following lemma.

Lemma 3.1 The differential equation of the developable surface

$$
\begin{gathered}
z=f(x, y) \text { is } r t-s^{2}=0 \text { where } \\
\mathrm{p}=\frac{\partial \mathrm{z}}{\partial \mathrm{x}}, \mathrm{q}=\frac{\partial \mathrm{z}}{\partial \mathrm{y}}, \mathrm{r}=\frac{\partial^{2} \mathrm{z}}{\partial \mathrm{x}^{2}}, \mathrm{~s}=\frac{\partial^{2} \mathrm{z}}{\partial \mathrm{x} \partial \mathrm{y}}, \mathrm{t}=\frac{\partial^{2} \mathrm{z}}{\partial y^{2}} \\
\text { Again, } K=\frac{e g-f^{2}}{E G-F^{2}}
\end{gathered}
$$

If we are given Monge type polynomial $\mathrm{z}=\mathrm{f}(\mathrm{x}, \mathrm{y})$ then

$$
\begin{aligned}
& \text { eg }-f^{2}=f_{x x} f_{y y}-f_{x y}^{2}=r t-s^{2}=0 \\
& \text { where } r=f_{x x}, t=f_{y y}, s=f_{x y}
\end{aligned}
$$


Example 3.1 Given the surface

$$
z=a+b x+c y+\sum_{n=2}^{N} a_{n}(p x+q y)^{n}
$$

where the coefficients of $\mathrm{x}$ and $\mathrm{y}$ are all constants. Is this surface developable?

$$
\begin{aligned}
z_{x}= & b+\sum_{n=2}^{N} a_{n} n p(p x+q y)^{n-1} \\
z_{x x}= & \sum_{n=2}^{N} a_{n} n(n-1) p^{2}(p x+q y)^{n-2}=r \\
z_{x y}= & \sum_{n=2}^{N} a_{n} n(n-1) p q(p x+q y)^{n-2}=s \\
z_{y}= & c+\sum_{n=2}^{N} a_{n} n q(p x+q y)^{n-1} \\
z_{y y}= & \sum_{n=2}^{N} a_{n} n(n-1) q^{2}(p x+q y)^{n-2}=t \\
e g- & f^{2}=z_{x x} z_{y y}-z_{x y}^{2}=r t-s^{2} \\
& -p^{2} q^{2}\left(\sum_{n=2}^{N} a_{n} n(n-1)(p x+q y)^{n-2}\right)^{2} \\
= & 0 \\
= & p^{2} q^{2}\left(\sum_{n=2}^{N} a_{n} n(n-1)(p x+q y)^{n-2}\right)^{2} \\
& \\
&
\end{aligned}
$$

Equation (3.15) demonstrates that the surface is developable.

If the rectangular coordinates $\mathrm{x}, \mathrm{y}, \mathrm{z}$ are the function of two independent variable $\mathrm{u}, \mathrm{v}$, then we can express surface $r=r(u, v)=r(x, y, z)$, where $\mathrm{x}=\mathrm{x}(\mathrm{u}, \mathrm{v}), \mathrm{y}=\mathrm{y}(\mathrm{u}, \mathrm{v}), \mathrm{z}=\mathrm{z}(\mathrm{u}, \mathrm{v})$. If we cancel $(\mathrm{u}, \mathrm{v})$ then we get equation $F(x, y, z)=0$. On the other hand, if point $(x, y, z)$ satisfies $F(x, y, z)=0$ and $\frac{\partial \mathrm{F}}{\partial \mathrm{z}} \neq 0$, then let $x=u, y=v$, and we can solve $\mathrm{z}$ and obtain $x=u, y=v, z=f(u, v)$. The next theorem gives us another form of developable surface of $F(x, y, z)=0$. During the derivation of this theorem, we can apply the property of the lemma, $r t-s^{2}=0$. In other words, $F_{x x} F_{y y}-F_{x y}^{2}=0$, for an implicit function defined by the surface $F(x, y, z)=0$, the Gaussian curvature can be expressed in terms of the gradient vector $\nabla F$ and Hessian matrix $\mathrm{H}(\mathrm{F})$. 


$$
K=\frac{\left|\begin{array}{cc}
H(F) & \nabla F^{t} \\
\nabla F & 0
\end{array}\right|}{|\nabla F|^{4}}=\frac{\left|\begin{array}{llll}
F_{x x} & F_{x y} & F_{x z} & F_{x} \\
F_{x y} & F_{y y} & F_{y z} & F_{y} \\
F_{x z} & F_{y z} & F_{z z} & F_{z} \\
F_{x} & F_{y} & F_{z} & 0
\end{array}\right|}{|\nabla F|^{4}}
$$

Theorem 3.3 Shows that a surface $F(x, y, z)=0$ is developable if

$$
\begin{aligned}
& \left|\begin{array}{llll}
F_{x x} & F_{x y} & F_{x z} & F_{x} \\
F_{x y} & F_{y y} & F_{y z} & F_{y} \\
F_{x z} & F_{y z} & F_{z z} & F_{z} \\
F_{x} & F_{y} & F_{z} & 0
\end{array}\right|=0 \text { where } \mathrm{F}_{x}=\frac{\partial F}{\partial x} \\
& \left|\begin{array}{llll}
F_{x x} & F_{x y} & F_{x z} & F_{x} \\
F_{x y} & F_{y y} & F_{y z} & F_{y} \\
F_{x z} & F_{y z} & F_{z z} & F_{z} \\
F_{x} & F_{y} & F_{z} & 0
\end{array}\right|=\left|\begin{array}{ccc}
F_{x x} F_{y y}-F_{x y}^{2} & F_{x x} F_{y z}-F_{x z} F_{x y} & F_{x x} F_{y}-F_{x} F_{x y} \\
F_{x x} F_{y z}-F_{x y} F_{x z} & F_{x x} F_{z z}-F_{x z}^{2} & F_{x x} F_{z}-F_{x} F_{x z} \\
F_{x x} F_{y}-F_{x} F_{x y} & F_{x x} F_{z}-F_{x z} F_{x} & -F_{x}^{2}
\end{array}\right| \\
& =\left|\begin{array}{ccc}
-\left(F_{x x} F_{y z}-F_{x y} F_{x z}\right)\left(F_{x x} F_{y z}-F_{x z} F_{x y}\right) & -\left(F_{x x} F_{y z}-F_{x y} F_{x z}\right)\left(F_{x x} F_{y}-F_{x} F_{x y}\right) \\
-\left(F_{x x} F_{y}-F_{x} F_{x y}\right)\left(F_{x x} F_{y z}-F_{x z} F_{x y}\right) & -\left(F_{x x} F_{y}-F_{x} F_{x y}\right)\left(F_{x x} F_{y}-F_{x} F_{x y}\right)
\end{array}\right| \\
& =\left(F_{x x} F_{y z}-F_{x y} F_{x z}\right)\left(F_{x x} F_{y z}-F_{x z} F_{x y}\right)\left(F_{x x} F_{y}-F_{x} F_{x y}\right)\left(F_{x x} F_{y}-F_{x} F_{x y}\right) \\
& -\left(F_{x x} F_{y}-F_{x} F_{x y}\right)\left(F_{x x} F_{y z}-F_{x z} F_{x y}\right)\left(F_{x x} F_{y z}-F_{x y} F_{x z}\right)\left(F_{x x} F_{y}-F_{x} F_{x y}\right) \\
& =0
\end{aligned}
$$

The advantage of this matrix form is that it is easy to extend to n-dimensional functions. For example, if the given implicit function is $F\left(x_{1}, x_{2}, \ldots x_{n}\right)=0$ then the Hessian matrix $\mathrm{H}(\mathrm{F})$ is of order

nxn, whose elements are second-order partial derivative, plus one column or row of the gradient vector $\nabla F$. Hence the numerator of Gaussian Curvature turns out to be an $(n+1)(n+1)$ matrix.

We now repeat the previous example by moving term $\mathrm{z}$ to the right side and write

$$
\begin{aligned}
& F(x, y, z)=a+b x+c y+\sum_{n=2}^{N} a_{n}(p x+q y)^{n}-z \\
& F_{x}=Z_{x}, \quad F_{y}=Z_{y}, \quad F_{z}=-1, \\
& F_{x x}=Z_{x x}=r, \quad F_{x y}=Z_{x y}=F_{y x}=s, \\
& F_{y y}=Z_{y y}=t, \quad F_{x z}=0, F_{y z}=0, F_{z z}=0,
\end{aligned}
$$

Substituting the results of (3.18) in matrix (3.17)

yields $F_{x x} F_{y y}-F_{x y}^{2}=0$, that is required for any developable surface. 


$$
\left|\begin{array}{cccc}
F_{x x} & F_{x y} & 0 & F_{x} \\
F_{x y} & F_{y y} & 0 & F_{y} \\
0 & 0 & 0 & -1 \\
F_{x} & F_{y} & -1 & 0
\end{array}\right|=F_{x x} F_{y y}-F_{x y}^{2}=0 .
$$

\section{Concluding Remark}

Experimental data usually can be fitted by a multiple linear regression model. Suppose that the response $\mathrm{y}$ is related to $\mathrm{n}$ covariates, also known as explanatory variables, regressors, or predictor $x_{1}, x_{2}, \ldots x_{n}$ is in a linear functional form. Statisticians call this regression analysis. Mathematically speaking, we fit the data in a hyper-plane that is the plane in n-dimensional space. Since the Hessian matrix has all second-order partial derivatives, the elements in that matrix are all zero. This means that the determinant of Gaussian Curvature equals zero. In this sense, we can be sure that the multiple linear regression analysis, in general, has no information loss problem. However, from Example 2.1 we see that Gaussian curvature is different from zero. Therefore, there is information loss. It is natural to ask how much information was lost due to the use of that model. Efron (1975) considers arbitrary one-parameter families and quantifies how nearly "exponential" they are. A quantity , $\gamma(\theta)$, called "the statistical curvature" at $\theta$ is introduced such that $\gamma(\theta)$ is identically zero if the model is exponential, and greater than zero, for at least some $\theta$ values otherwise. Efron

shows that the models with small curvature enjoy nearly the good statistical properties of exponential families. Large curvatures indicate a breakdown of this favorable situation. We adopt his fundamental proposition and extend it to $\mathrm{n}$-dimensional space. If $\mathrm{n}$-dimensional Gaussian curvature equals zero, we can be sure there is no information loss. However, if the Gaussian curvature in n-dimensional space is a positive number, then the smaller curvature is better than the larger one. In other words, the smaller Gaussian curvature causes less information loss.

\section{References}

Balakrishnan, N., \& Chen, W. W. S. (1997). CRC Handbook of Order Statistics from Inverse Gaussian Distributions with Applications. CRC Press, Baca raton.

Chen, W. W. S. (2016). On the comparison of statistical Curvature with Gaussian curvature. International Journal of Mathematics and Statistics Studies, 4(6), 13-23, December.

Chen, W. W. S., \& Kotz, S. (2013). The Riemannian structure of the Three-Parameter Gamma Distribution. Applied Mathematics, 4(3).

Efron, B. (1975). Defining the curvature of a statistical problem (with application to second order efficiency). The Annals of Statistics, 3(6), 1, 189-1242.

Fisher, R. A. (1925). Theory of statistical estimation. Proc, Cambridge Philos. Soc. 122, 700-725.

Fuller, W. E. (1914). Flood flows. Transactions of the American Society of Civil Engineers. 77, 564.

Grey, A. (1993). Modern Differential Geometry of Curves and Surfaces. CRC Press, Inc. Boca Raton.

Griffith, A. A. (1920). The phenomena of rupture and flow in solids. Philosophical Transactions of the Royal Society of London, Series A, 221, 163-198.

Gumbel, E. J. (1958). Statistics of Extremes, New York. Columbia University Press.

Johnson, N. L., Kotz, S., \& Balakrishnan, N. (1995). Continuous Univariate Distributions Volume 2, second edition. John Wiley \& Sons, Inc.

Kass, R. E., \& Vos, P. W. (1997). Geometrical Foundations of Asymptotic Inference. John Wiley \& Sons, Inc.

Kass, R. E. (1984). Canonical parameterizations and zero parameter-effects curvature. J. Roy. Statist. Soc. B46, 1, 86-92.

Rao, C. R. (1962). Efficient estimates and optimum inference procedures in large samples. J. Roy. Statist. Soc. Ser. B. 24, 46-72.

Rao, C. R. (1963). Criteria of estimation in large samples. Sankhya, 25, 189-206.

Rao, C. R. (1961). Asymptotic efficiency and limiting information. (J. Neyman. Ed.) Proc. Fourth Berkeley Symp. Math. Statist. Prob. 1, 531-545. Univ. of California Press.

Seshadri, V. (1993). The Inverse Gaussian Distribution: A Case Study in Exponential families. Clarendon Press, Oxford, England.

Struik, D. J. (1961). Lectures on Classical Differential Geometry. Second Edition, Dover Publications, Inc. 
Tweedie, M. C. K. (1945). Inverse statistical variates. Nature, 155, 453.

Villarroya, A., \& Oller, J. M. (1991). Statistical Tests for the Inverse Gaussian Distribution Based on Rao Distance. Barcelona : University of Barcelona, Department of Statistics.

\section{Appendix I}

The following theorem can be found in most standard textbook.

Theorem: Let $x: \mathrm{U} \rightarrow \mathrm{R}^{3}$ be a regular patch. Then the Gaussian curvature and mean curvature of $\mathrm{x}$ are given by the formulas

$$
K=\frac{e g-f^{2}}{E G-F^{2}}, \quad H=\frac{e G-2 f F+g E}{2\left(E G-F^{2}\right)}
$$

Where $\mathrm{e}, \mathrm{f}$ and $\mathrm{g}$ are the coefficients of the second fundamental form relative to $\mathrm{x}$ and $\mathrm{E}, \mathrm{F}$, and $\mathrm{G}$ are the coefficients of the first fundamental form.

Proof. We compute $\mathrm{K}$ and $\mathrm{H}$ using the basis $\left\langle x_{u}, x_{v}\right\rangle$. From the Weingarten Equations we get

$$
\begin{gathered}
K=\operatorname{det}\left|\begin{array}{rr}
-\frac{f F-e G}{E G-F^{2}} & -\frac{e F-f E}{E G-F^{2}} \\
-\frac{g F-f G}{E G-F^{2}} & -\frac{f F-g E}{E G-F^{2}}
\end{array}\right|=\frac{(f F-e G)(f F-g E)-(e F-f E)(g F-f G)}{\left(E G-F^{2}\right)^{2}}=\frac{e g-f^{2}}{E G-F^{2}} \\
H=\frac{1}{2} t r\left|\begin{array}{cc}
-\frac{f F-e G}{E G-F^{2}} & -\frac{e F-f E}{E G-F^{2}} \\
-\frac{g F-f G}{E G-F^{2}} & -\frac{f F-g E}{E G-F^{2}}
\end{array}\right|=\frac{-1}{2} \frac{(f F-e G)+(f F-g E)}{E G-F^{2}} \\
=\frac{e G-2 f F+g E}{2\left(E G-F^{2}\right)}
\end{gathered}
$$

\section{Copyrights}

Copyright for this article is retained by the author(s), with first publication rights granted to the journal.

This is an open-access article distributed under the terms and conditions of the Creative Commons Attribution license (http://creativecommons.org/licenses/by/4.0/). 